\title{
ECO - Comunicação síncrona para Matemática e Física
}

\author{
Vinícius Teixeira da Silva \\ Marcus Vinicius de Azevedo Basso \\ Instituto de Matemática - Universidade Federal do Rio Grande do Sul \\ vinicius@mat.ufrgs.br, mbasso@ufrgs.br
}

\begin{abstract}
Resumo
Ao longo dos últimos anos profissionais da área de ensino atentaram para o potencial da Internet como um importante meio de comunicação e de aprendizagem. No caso das ciências exatas, um obstáculo logo se colocou: a dificuldade de escrever expressões matemáticas e expressões envolvendo símbolos próprios de cada área, como a Matemática e a Física. Esse trabalho visa o desenvolvimento de uma alternativa para oferecer uma primeira solução para professores e alunos de Matemática e Física. Se trata de um chat com um editor de expressões matemáticas e que permite upload de imagens que podem ser inseridas no próprio corpo da conversação online. Desenvolvido em PHP e JavaScript, com características open source, o ECO possibilita futuras contribuições de outros grupos de pesquisa.
\end{abstract}

Palavras-chave: chat para matemática, educação à distância.

Title: ECO - Synchronous communication for Mathematics and Physics

\begin{abstract}
Through the last years professionals of the educational area had attempted to the Internet's potential as an important medium of communication and learning. In the case of mathematic sciences an obstacle was placed soon: the difficulty to write mathematics' expressions and expressions that uses restricted symbols of some areas as Mathematics and Physics. This work aims the development of an alternative that offers a first solution for teachers and students of Mathematics and Physics. We present one chat that allows the edition of mathematics' expressions and the upload of images that can be inserted in the online conversation itself. Developed in PHP and Javascript, with characteristics of an open source, ECO enables future contributions of other groups of research.
\end{abstract}

Keywords: chat for mathematics, e-learning.

\section{Introdução}

A comunicação com o uso da informática evoluiu muito desde a criação desta ciência. O computador como ferramenta multimídia tem transcendido de útil para indispensável para qualquer área do conhecimento, em particular para as ciências exatas.

\footnotetext{
${ }^{1}$ Trabalho vinculado ao RIVED - Secretaria de Educação a Distância - Ministério da Educação, desenvolvido pelo Acadêmico Vinícius Teixeira da Silva sob orientação do Prof. Dr. Marcus Vinicius de Azevedo Basso. 
Com os atuais incentivos do nosso governo aos projetos de educação a distância é inevitável que nossas universidades cada vez mais precisem de instrumentos para propagar e sustentar seus cursos em todos os níveis via Internet.

Porém, tentemos utilizar uma sala de bate-papo ou um programa de conversação síncrona como os disponíveis para conversar a respeito da resolução de um problema matemático, onde se faça necessário apresentar uma expressão com símbolos diferentes daqueles que temos nos teclados.

É claro que ao olharmos para um teclado facilmente perceberemos que ali faltam muitos dos símbolos utilizados para construir expressões matemáticas.

Alunos e professores adotam as mais variadas estratégias para driblar as dificuldades na elaboração de textos onde expressões matemáticas são necessárias. Uma das soluções é escrever utilizando notação de programas editores de planilhas ou notação de linguagens de programação. Por exemplo, na cadeira de Ensino de Matemática I do Curso de Licenciatura em Matemática da Universidade Federal do Rio Grande do Sul, onde os alunos devem utilizar o computador para desenvolverem suas tarefas de aula, uma primeira pergunta que os estudantes fazem é: "Como eu escrevo a raiz quadrada?". Essa ausência impõe o uso da própria expressão "raiz quadrada" ou o que é comumente utilizado em aplicativos de Matemática, SQRT [parâmetro].

Mesmo expressões mais simples, como a que envolve a utilização de uma potência ou um índice, e que para ser resolvido tem-se que apelar para o uso de código HTML, tornam o trabalho mais difícil e desviam o foco do próprio trabalho que é o de pensar na solução da questão.

Outra estratégia é resolver exercícios manualmente e digitalizar as soluções para posterior envio através de mensagem eletrônica - o que atrasa o encaminhamento da interação entre alunos/alunos ou alunos/professores e a própria discussão matemática ali envolvida. Vemos abaixo uma aluna (Figura 1) do Curso de Licenciatura em Matemática da Universidade Federal do Rio Grande do Sul digitalizando um texto com expressões matemáticas para discutir o assunto com colegas via e-mail.

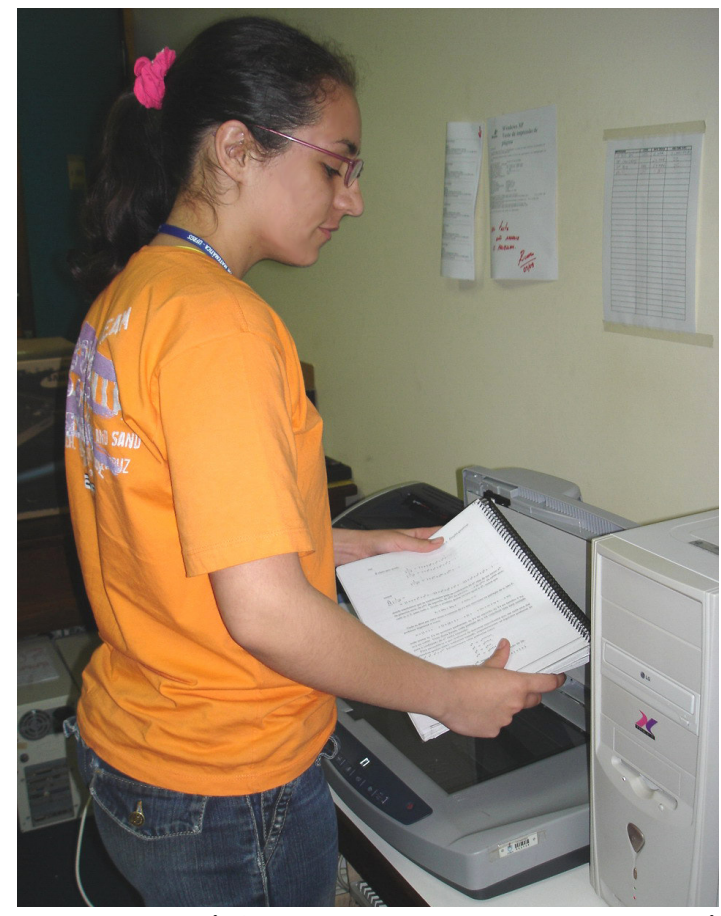

Figura 1 - Uma das estratégias para o estudo de Matemática a distância 
As questões acima nos remetem a pensarmos sobre quais são as carências na educação matemática a distância.

As habilidades para utilizar um conjunto de programas com finalidades distintas (editores de texto, editores de fórmulas, mailers, softwares para digitalização de imagens, etc) exigem tempo e disposição de aprendizagem por parte dos usuários, professores e alunos.

A partir da orientação dos coordenadores do projeto ao qual estamos vinculados e das constantes discussões sobre a facilitação do atendimento extra-classe com recursos computacionais ficou estabelecida como meta a produção de uma ferramenta inovadora que pudesse dar conta das demandas já relatadas.

Assim, o objetivo do projeto foi oferecer uma solução para os problemas impostos à Educação de Ciências Exatas a Distância, no que se refere à edição de expressões matemáticas. Para isso, desenvolvemos e continuamos aperfeiçoando uma ferramenta para comunicação digital que deverá unificar as funções dos programas anteriores (mailers, digitalizadores, editores de fórmulas, etc). Tal ferramenta chama-se ECO Editor Científico On Line.

\section{Descrição da ferramenta}

O ECO foi desenvolvido em PHP e JavaScript. Os componentes da ferramenta são: um ambiente de conversação (PHP), e um editor HTML que permite inserção de símbolos matemáticos (JavaScript), formatação de texto e inserção de imagens.

O editor - que diferencia o ECO das demais salas de bate-papo - foi baseado no editor HTML WYSIWYG Editor, originalmente da OpenWebWare, cujo código fonte é livre.

Na versão original, são disponibilizadas ferramentas de formatação de texto, inserção de imagens e tabelas e visualização nos modos texto ou HTML. O WYSIWYG Editor transforma uma simples área de texto de formulário HTML em um eficiente editor. Para o ECO, aproveitamos a maioria das ferramentas de formatação, traduzimos as janelas do WYSIWYG Editor e acrescentamos as barras de ferramentas cujos botões inserem símbolos matemáticos. Adicionamos também alguns botões com estruturas de expressões matemáticas predefinidas como integral, somatório, produtório e fração. $\mathrm{O}$ usuário pode compor integrais, frações, integral de funções com forma fracionária, etc. tudo apenas usando o procedimento de clicar e arrastar, como nos editores de texto que a maioria dos usuários conhecem, alguns deles disponíveis gratuitamente na Web.

Inserção de imagens nas conversações também é possível, desde que a imagem esteja em qualquer site da Web. O ECO também permite que o usuário faça upload de imagens. Além de inserir a imagem, também se pode alterar as dimensões, atribuir bordas, definir espaçamentos e layout da imagem em relação ao texto.

Quando temos pessoas interagindo através da nossa ferramenta, evitamos a incompatibilidade entre os diferentes softwares que as pessoas fazem uso, isto é, se dois usuários utilizam editores de fórmulas e textos distintos pode haver dificuldades na leitura dos arquivos um do outro, o que não ocorreria se ambos utilizassem a mesma ferramenta de edição e comunicação. Além disso, graças a portabilidade do ECO, usuários do Microsoft Windows podem comunicar-se sem nenhuma dificuldade com usuários do Linux ou MAC-OS, desde que optem por navegadores que sejam compatíveis com JavaScript.

Outra vantagem é a dispensabilidade de instalação: basta os usuários entrarem em um chat, o próprio ECO, neste caso, e utilizarem os recursos que ele disponibiliza.

Em resumo, os usuários da nossa ferramenta podem:

- Estabelecer comunicação via texto de maneira síncrona 
- Inserir imagens e exibi-las na área de interação

- Editar e formatar textos e expressões matemáticas e exibir o resultado na área de interação

A aparência do ECO, na parte que diz respeito à edição (Figura 2), apresenta ferramentas com ícones similares aos dos principais editores de textos - o que facilita sua utilização.

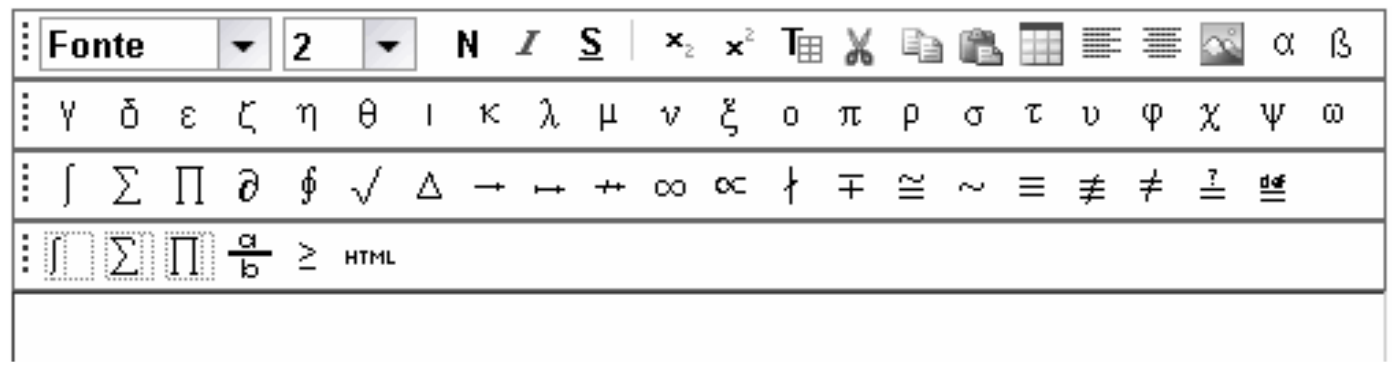

Figura 2 - Aparência do editor do ECO

A possibilidade de compor expressões com um simples arrastar de mouse (Figura 3) é uma das vantagens do uso da nossa ferramenta:

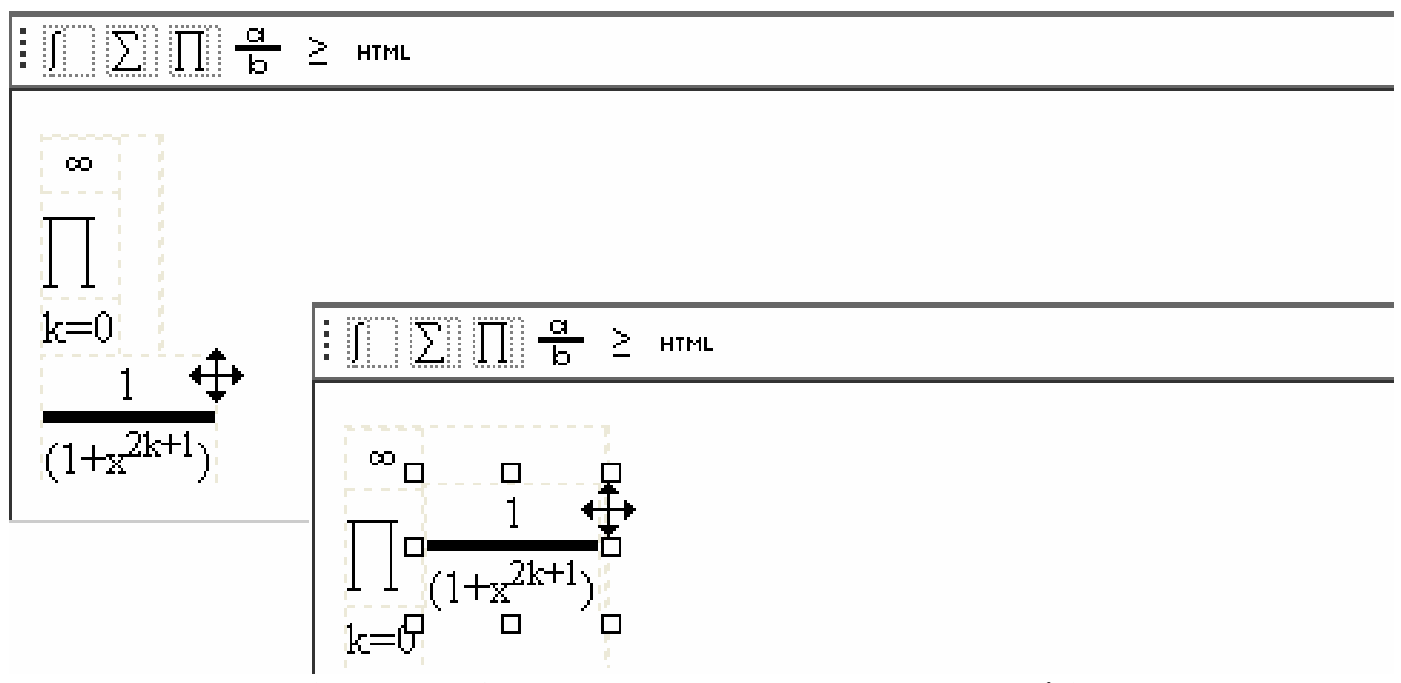

Figura 3 - Compondo uma expressão matemática

Existem esforços para produzir ferramentas que utilizam Mathml para sanar as dificuldades da educação a distância de ciências exatas. A complexidade dos códigos foi um dos motivos pelo qual optamos por não utilizar Mathml. O outro motivo foi a urgência de apresentar uma solução, ainda que provisória, para a comunicação entre usuários que, em seus textos, utilizam expressões matemáticas. Assim, nossa ferramenta não utiliza Mathml. Ela cria textos formatados a partir de tags HTML, com a possibilidade de inserir caracteres Unicode (Figura 4): 


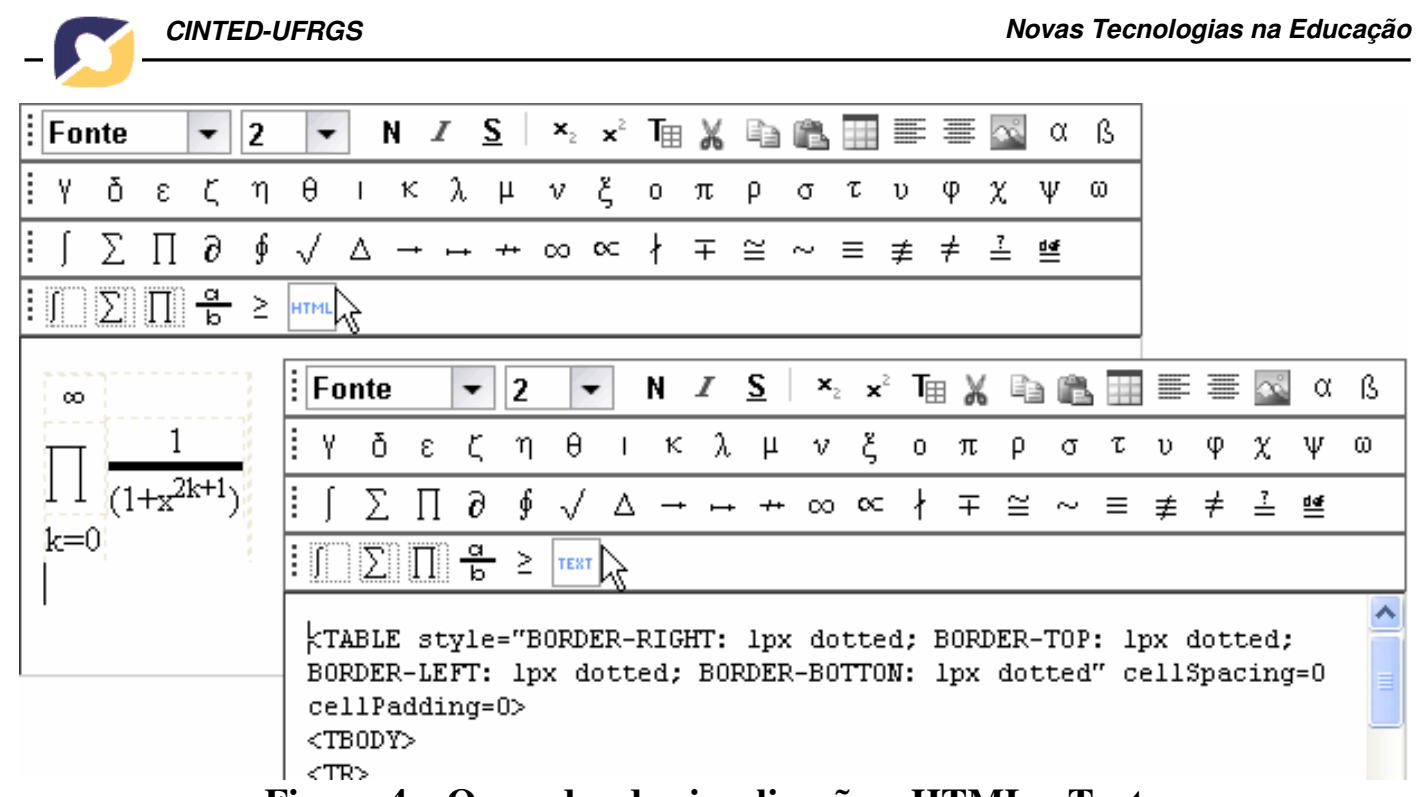

Figura 4 - Os modos de visualização - HTML e Texto

O funcionamento é bem simples: o usuário acessa uma página onde um formulário armazena seu nome ou nickname em uma plataforma de chat desenvolvida em PHP e utiliza essa plataforma para interagir com os presentes. É neste cliente de chat que o usuário experimenta a facilidade de escrever textos e expressões matemáticas.

Após enviar o que fora escrito em um campo de texto, a mensagem é processada pelo servidor e ecoada para todos os clientes.

Além de permitir a escrita de textos e expressões matemáticas, o ECO também permite o envio de imagens que são inseridas na própria área da conversação online. Na figura 5 apresentamos um trecho de conversação no qual estão inseridas imagens utilizadas como auxiliares em uma discussão sobre geometria.
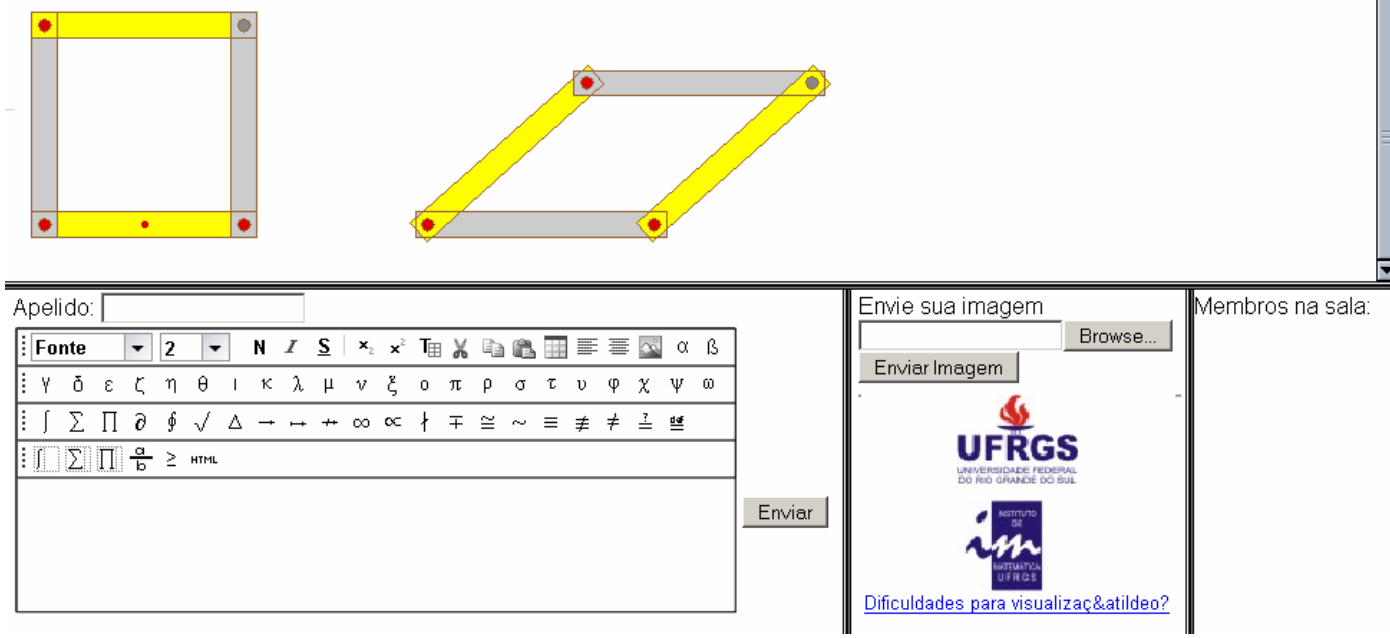

Figura 5 - Upload e visualização de imagens na área de conversação

Em termos de perspectivas, pretende-se aperfeiçoar a versão atual visando possibilitar a comunicação com expressões na área da Química. Além disso, o caráter open source que atribuímos ao nosso software irá permitir contribuições de outros programadores para sua melhora e a possível implementação do mesmo para o desenvolvimento de ferramentas para outras áreas do conhecimento. 


\section{Conclusões}

Utilizando o ECO, quais as conseqüências do que foi exposto para a educação de ciências exatas a distância? Primeiramente, o aluno/professor não precisará ter conhecimentos sobre diversos programas. Isto significa poupar tempo em treinamento de profissionais nas universidades, na dispensabilidade de inserir forçadamente hábitos nos alunos e professores de uma área que não são da informática.

Outra vantagem de não produzir expressões matemáticas em softwares diversos é a garantia de que não haverá incompatibilidade de leitura de arquivos nos computadores dos participantes da interação.

Tudo isto tem sido altamente excludente, ou seja, um usuário determina qual software será utilizado e os outros devem tentar instalar o programa nos seus computadores. Mas todos possuem computadores capazes de rodar qualquer software? Um último obstáculo que vemos sendo transposto é o tempo. Escrever uma fórmula, o passo a passo de uma derivação de função ou a explicação de um conceito de análise requer tempo; enviar o que foi produzido e esperar o retorno, é óbvio que requer, novamente, mais tempo. Aliás, dentre outros, o atraso no envio e recebimento das informações é um dos principais motivos do desinteresse do aluno em obter ajuda a distância. Com o ECO e o conceito de comunicação síncrona que este proporciona, temos um melhor aproveitamento do tempo, explorando possibilidades de sanar dúvidas na medida em que se esteja desenrolando um diálogo em linguagem matemática bem como na utilização de imagens que permitam esclarecer aspectos relacionados tanto à Geometria quanto a outras áreas de Matemática.

\section{Referências}

Wood, A. (1999) Unicode and Multilingual Support in HTML, Fonts, Web Browsers and Other Applications. In: <http://www.alanwood.net/ unicode/\#links>. Acesso em 15 mar. 2005.

Documentação do WYSIWYG Editor. In:

$<$ http://www.openwebware.com/products/openwysiwyg/docs/>. Acesso em 20 jun. 2006. 